\title{
Hybrid quantum anomalous Hall effect at graphene-oxide interfaces
}

\author{
Z. Zanolli, ${ }^{1,2,{ }^{*}}$ C. Niu, ${ }^{3}$ G. Bihlmayer,${ }^{3}$ Y. Mokrousov,${ }^{3,4}$ P. Mavropoulos, ${ }^{3}$ M. J. Verstraete, ${ }^{5}$ and S. Blügel ${ }^{3}$ \\ ${ }^{1}$ Catalan Institute of Nanoscience and Nanotechnology (ICN2), CSIC and BIST, Campus UAB, Bellaterra, 08193 Barcelona, Spain \\ ${ }^{2}$ Institute for Theoretical Solid State Physics and European Theoretical Spectroscopy Facility (ETSF), \\ RWTH Aachen University, D-52056 Aachen, Germany \\ ${ }^{3}$ Peter Grünberg Institut (PGI-1) and Institute for Advanced Simulation (IAS-1), \\ Forschungszentrum Jülich and JARA, D-52425 Jülich, Germany \\ ${ }^{4}$ Institute of Physics, Johannes Gutenberg University Mainz, 55099 Mainz, Germany \\ ${ }^{5}$ Nanomat/Qmat/CESAM and European Theoretical Spectroscopy Facility (ETSF), Université de Liège, B-4000 Sart Tilman, Belgium
}

(Received 6 June 2018; published 5 October 2018)

\begin{abstract}
Interfaces are ubiquitous in materials science, and in devices in particular. As device dimensions are constantly shrinking, understanding the physical properties emerging at interfaces is crucial to exploit them for applications, here for spintronics. Using first-principles techniques and Monte Carlo simulations, we investigate the mutual magnetic interaction at the interface between graphene and an antiferromagnetic semiconductor $\mathrm{BaMnO}_{3}$. We find that graphene deeply affects the magnetic state of the substrate, down to several layers below the interface, by inducing an overall magnetic softening, and switching the in-plane magnetic ordering from antiferromagnetic to ferromagnetic. The graphene- $\mathrm{BaMnO}_{3}$ system presents a Rashba gap 300 times larger than in pristine graphene, leading to a flavor of quantum anomalous Hall effect (QAHE), a hybrid QAHE, characterized by the coexistence of metallic and topological insulating states. These findings could be exploited to fabricate devices that use graphene to control the magnetic configuration of a substrate.
\end{abstract}

DOI: 10.1103/PhysRevB.98.155404

\section{INTRODUCTION}

The interface between materials can be considered as the ultimate spintronics device, not only in terms of miniaturization but also to unlock unique design possibilities and new physical properties which are unattainable in the individual bulk materials [1,2]. Emergent interfacial properties induced by spin-orbit coupling (SOC) include Rashba-Edelstein spinto-charge conversion and spin-momentum locking at the surface of topological insulators. Interfaces present a natural breaking of spatial inversion symmetry, resulting in a Rashba SO splitting of the electronic bands which is, in general, higher than in bulk. In particular, SO effects at oxide interfaces are crucial for low-power spintronics applications thanks to the expected long carrier lifetime and high Rashba coefficient [3]. Carbon-based nanomaterials are prime candidates for spin-based devices, thanks to their long spin coherence length (up to $10^{4} \mathrm{ps}$ ) and high Fermi velocity [4]. However, a pristine hexagonal network of carbon is not magnetic, controlled injection of spin polarization in graphene or carbon nanotubes is far from trivial [5], and SOC in C is too small for practical applications [6]. Possible approaches are to cut the graphene in nanoribbons, introduce defects [7], dope with atoms [8] or nanoclusters [9] possessing higher spin-orbit coupling. Magnetism can be induced in graphene by proximity interaction with a magnetic insulating substrate, as predicted by first-principles calculations $[10,11]$ and recently proven experimentally [12,13], or at graphene/topological insulator interfaces [14]. Spin-valley locking is found in heterostruc-

*zeilazanolli@gmail.com tures of graphene with transition-metal dichalcogenides $[12,15,16]$.

In this work, we invert the approach, and propose to use graphene to control the magnetic properties of an insulating substrate, using as a prototype antiferromagnetic (AFM) $\mathrm{BaMnO}_{3}$. Several transition-metal (Mn, Sc, Ti, V, Fe) adatoms on graphene have been shown to induce the opening of a nontrivial gap and lead to the quantum anomalous Hall (QAH) effect [17]. Among the many possible transition-metal-based materials, $\mathrm{BaMnO}_{3}$ stands out as being insulating, magnetic, and close to lattice matched to a $4 \times 4$ graphene supercell. Through first-principles simulations including SOC we show that graphene changes strongly the substrate magnetization. The breaking of mirror symmetry in the hybrid system results in a Rashba spin-orbit gap more than 300 times that of pristine graphene $(24 \mu \mathrm{eV})$ [18], a topologically nontrivial band structure, and QAH conductivity. Graphene induces an overall magnetic softening, and even switches the magnetic ordering in the surface Mn layers. It is remarkable that the influence of graphene extends several unit cells into the magnetic substrate, an effect which has not been seen before [19]. Such structures, arising from an organic-magnetic semiconducting system, could find applications as a magnetic storage unit (memory) or spin filter. We demonstrate the coexistence of metallic and QAH states, contributing to a hybrid QAH effect and opening new perspectives for robust spin-orbit torques at higher temperatures.

\section{SPIN SPIRALS IN $\mathrm{BaMnO}_{3}$}

Bulk $\mathrm{BaMnO}_{3}$ is a magnetoelectric multiferroic with a ferroelectric ground state, as shown by collinear-spin 

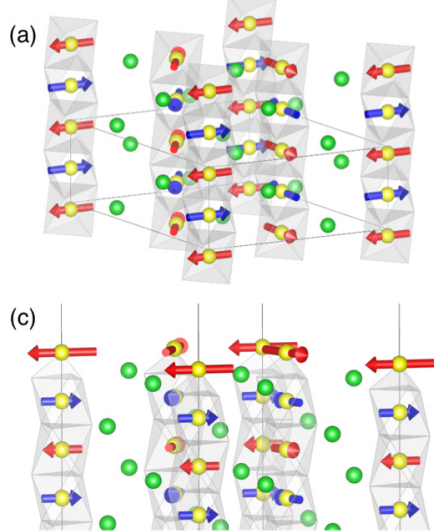

(e)

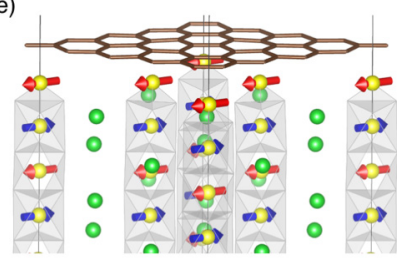

(g)

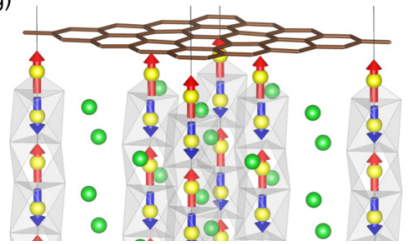

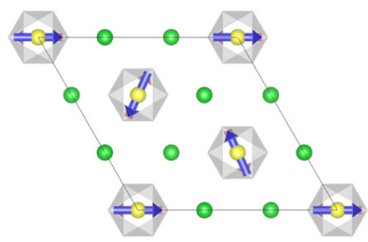

(d)

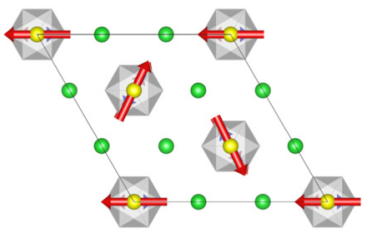

(f)

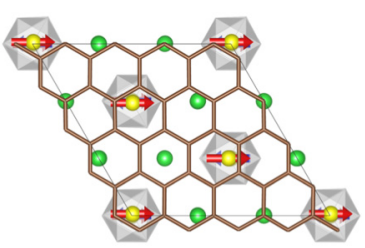

(h)

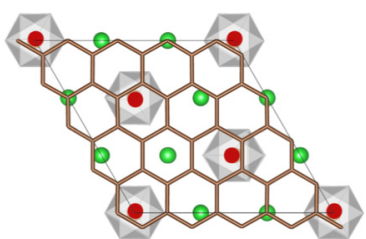

FIG. 1. Spin texture in real space for $\mathrm{BaMnO}_{3}$ bulk (a), (b) and slab without (c), (d) and with graphene (e)-(h), in side and top views. Red and blue colors indicate the spin directions in subsequent $\mathrm{Mn}$ layers. AFM coupling between layers is predicted in all cases. The magnetization in the bulk and slab without graphene has an easy plane perpendicular to [0001], characterized by anticlockwise spin spirals (b), (d). The interface with graphene results in a FM alignment in the surface, with the in-plane configuration (e), (f) still slightly favored with respect to the out-of-plane one $(\mathrm{g}),(\mathrm{h})$.

first-principles calculations [10] and neutron powder diffraction experiments [20]. The bulk primitive cell consists of two $\sqrt{3} \times \sqrt{3} \mathrm{BaMnO}_{3}$ layers stacked along the [0001] axis. First-principles calculations including SOC performed with SIESTA [21] and FLEUR [22] codes predict an AFM ordering along the [0001] direction and in-plane magnetization for bulk $\mathrm{BaMnO}_{3}$. The spins form a triangular lattice, with vectors oriented $120^{\circ}$ with respect to nearest neighbors [Figs. 1(a) and 1(b)], in agreement with experiment [20]. SIESTA calculations find that the in-plane magnetization is more stable by $0.05 \mathrm{meV}$ per $\mathrm{Mn}$ atom, and that the magnetic moment on $\mathrm{Mn}$ atoms is $2.41 \mu_{B}$. Although the experimental lattice constant of $\mathrm{BaMnO}_{3}$ and graphene match, the relaxed DFT values present a difference of $\sim 2 \%$. In order to ensure that the induced spin polarization in graphene is exclusively due to the proximity to the magnetic insulating substrate, we chose to keep graphene relaxed and strain $\mathrm{BaMnO}_{3}$ instead. Straining the $\mathrm{BaMnO}_{3}$ in-plane lattice constant by $2 \%$ slightly favors the in-plane easy direction, now by $0.16 \mathrm{meV}$ per Mn atom. Strain does not modify strongly the magnetic moment $(M=$ $2.43 \mu_{B}$ ), and the ground-state magnetic ordering (AFM between layers, and triangular in plane) is unchanged (see
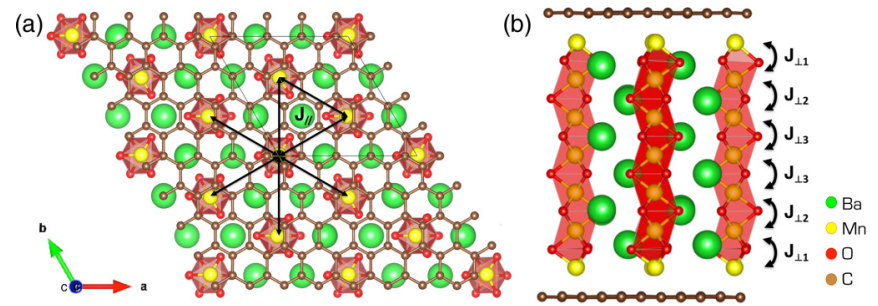

FIG. 2. Relaxed atomic structure for the graphene- $\mathrm{BaMnO}_{3}$ (gBMO) slab seen from the top (a) and from the side (b). Definition of the exchange coupling parameters between Mn atoms in plane $\left(J_{\|}\right)$ (a) and between planes $\left(J_{\perp}\right)$ (b) for the BMO and the gBMO slabs.

Table II). Both computational approaches predict a cycloidal in-plane spin spiral order for bulk $\mathrm{BaMnO}_{3}$. In ferroelectric materials with noncollinear magnetism, SOC can give rise to a Dzyaloshinskii-Moriya interaction (DMI) if the spin canting follows the Moriya rules [23]. The $120^{\circ}$ spin spiral in $\mathrm{BaMnO}_{3}$ rotates in plane, with a wave vector of $(1 \overline{1} 00)$, and DMI will favor one particular sense of rotation. A large DMI can also lead to a change of the easy plane of rotation. To evaluate the strength of the DMI, we calculate clockwise and anticlockwise rotating spin spirals along the (1100) direction, finding an energy difference of just $1 \mu \mathrm{eV}$ between the two spirals. A $2 \%$ in-plane strain of $\mathrm{BaMnO}_{3}$ enhances the macroscopic polarization [10] $\mathbf{P}$ from $0.267 \mu \mathrm{C} / \mathrm{cm}^{2}$ to $19.04 \mu \mathrm{C} / \mathrm{cm}^{2}$. This increases the strength of the DMI by a factor of 4 but is not so large as to influence the magnetic ground state.

\section{MAGNETIC ORDERING AT THE INTERFACE}

The magnetic properties of large $\mathrm{BaMnO}_{3}$ slabs with and without graphene [Figs. 1(c)-1(h)] are computed with SIESTA for noncollinear spins including SOC (see the Appendix for computational details). The slabs are chosen to be symmetric (hence paraelectric) and consist of $7 \mathrm{Mn}$ and $6 \mathrm{BaO}_{3}$ layers. The in-plane lattice constant of $\mathrm{BaMnO}_{3}$ is strained to that of graphene, and all the atomic positions are relaxed. The magnetic moment induced on $\mathrm{C}$ atoms computed including SOC is the same as in the collinear-spin case [10]. The $\mathrm{BaMnO}_{3}$ slab has in-plane magnetic moments and AFM coupling along the [0001] direction [Figs. 1(c) and 1(d)], as in the bulk [Figs. 1(a) and 1(b)]. The in-plane magnetization is favored with respect to the out-of-plane case by $2.27 \mathrm{meV}$ per Mn atom, a value enhanced compared to the bulk due to the presence of the surface, and the resulting increased magnetic moment of surface atoms (reported in Table VI). We find a huge qualitative change for the graphene- $\mathrm{BaMnO}_{3}$ slab: at the interface, the surface layer of $\mathrm{Mn}$ atoms becomes ferromagnetically coupled, and presents many different states very close in energy, with spins either in or out of plane, as long as the AFM coupling between planes is guaranteed. The in-plane FM ground state [Figs. 1(e) and 1(f)] for the surface layer is only $0.17 \mathrm{meV}$ per $\mathrm{Mn}$ atom lower than a state with out-of-plane magnetization for all layers [Figs. 1(g) and 1(h)] The in-plane AFM coupling of bulk BMO has been softened almost to nothing at the interface. The magnetic ground state based on the orbital criterion (maximal orbital moment $\left\langle L^{2}\right\rangle$ ) suggests out-of-plane FM spins at the surface. These must 
TABLE I. The exchange coupling strengths between Mn atoms in plane $\left(J_{\|}\right)$and between planes $\left(J_{\perp}\right)$ for the $\mathrm{BaMnO}_{3}(\mathrm{BMO})$ and the graphene- $\mathrm{BaMnO}_{3}$ (gBMO) slabs. Units are meV. As the slabs are symmetric, we used one in-plane parameter for each of the four inequivalent layers $\left(J_{\| i}, i=1,2,3,4\right)$ and three parameters to model the interplane interaction $\left(J_{\perp i}, i=1,2,3\right)$. The subscripts label the layers starting from the surface $(i=1)$ to the middle $(i=4)$ of each slab. The fit of the Heisenberg model is performed on 10 different low-energy magnetic configurations listed in Table IV of Appendix.

\begin{tabular}{lcrccccc}
\hline \hline & $J_{\| 1}$ & \multicolumn{1}{c}{$J_{\| 2}$} & $J_{\| 3}$ & $J_{\| 4}$ & $J_{\perp 1}$ & $J_{\perp 2}$ & $J_{\perp 3}$ \\
\hline BMO & 20.60 & -4.75 & -18.55 & -3.26 & -125.21 & -169.45 & -164.53 \\
gBMO & 18.26 & 0.83 & -10.79 & -6.30 & -110.88 & -106.04 & -139.86 \\
\hline \hline
\end{tabular}

at some depth return to the bulk in-plane triangular lattice with AFM coupling. The spin-flop depth is governed by the balance between very strong interplane AFM coupling and the preference of the surface layers for FM in-plane coupling. Energy differences, magnetic moments, and orbital moments are reported in the Appendix (Tables V-IX). We will see below that this is consistent with the low-temperature Monte Carlo results, where the presence of graphene increases the out-of-plane component of the spin vectors at the interface. In this way, graphene can be used to control and tune the magnetism at the interface with a substrate.

\section{SPIN DYNAMICS AT FINITE TEMPERATURE}

In order to model the Mn spin dynamics, we combine collinear first-principles and Monte Carlo techniques. In the Appendix we detail the model and comment on the choice of spin configurations for the fitting procedure, as well as the exchange correlation effects [24-26].

In bulk $\mathrm{BaMnO}_{3}$, we find that the AFM coupling between planes is the dominant effect (Table III). The in-plane interaction is a frustrated AFM: the collinear spin model cannot reproduce the triangular alignment observed in SOC calculations and in experiment [20], but the resulting Heisenberg Hamiltonian is consistent with both. Both with and without graphene, the dominant mechanism in the slabs is the antiferromagnetic (AFM) coupling between planes: all the $J_{\perp}$ are negative and at least one order of magnitude larger than the $J_{\|}$, as in the bulk (Table I). Contrary to the bulk, both slabs favor FM coupling at the surface $\left(J_{\| 1}>0\right)$. For the bare slab, this is due to the two dangling electrons per Mn surface atom, which favor Mn spins perpendicular to the interface, visible in the projected density of states (see Appendix). When graphene is present, the strong covalent interaction between $\mathrm{C}$ $\pi$ and Mn $d$ states "pins" the spin density perpendicular to the surface [10]. The in-plane coupling $J_{\|}$is most affected by the presence of graphene: in general, the absolute value of $J_{\|}$is reduced, indicating a magnetic softening of the substrate due to graphene. Most strikingly, the graphene also changes the inplane magnetic ordering of the penultimate layer from AFM $\left(J_{\| 2}<0\right.$ without graphene and in bulk) to FM $\left(J_{\| 2}>0\right.$ with graphene). The central layers of both slabs always present an AFM in-plane coupling $\left(J_{\| 3}<0\right)$, consistent with bulk $\mathrm{BaMnO}_{3}$, which shows that the slab is thick enough.
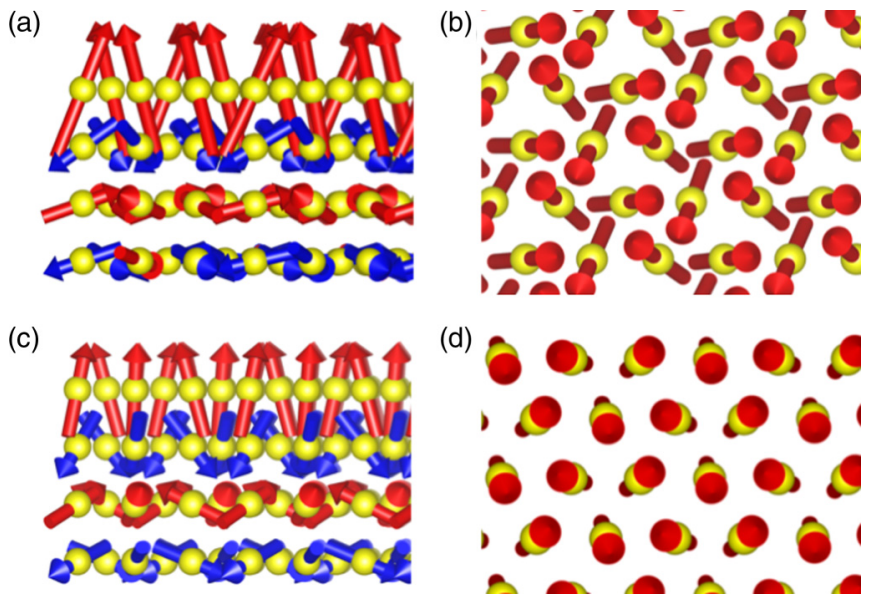

FIG. 3. Side (left panels) and top (right panels) views of the spins on Mn atoms (yellow spheres), obtained from Monte Carlo simulations at $5 \mathrm{~K}$ for the $\mathrm{BaMnO}_{3}$ slab without (top panels) and with (bottom panels) graphene. The presence of graphene changes the spin direction of the surface layer from mostly in plane to mostly out of plane. The other half of the slab is symmetric.

The exchange coupling parameters were used to obtain the thermodynamics and dynamical magnetic response of the BMO and gBMO systems. We perform Monte Carlo calculations using the Heisenberg Hamiltonian:

$$
H=-\frac{1}{2} \sum_{\alpha=1}^{7} \sum_{i \neq j} J_{\| \alpha} S_{i \alpha} S_{j \alpha}-\sum_{\alpha=1}^{6} \sum_{i} J_{\perp \alpha} S_{i \alpha} S_{i(\alpha+1)},
$$

where $S$ are the spin vectors on the individual Mn atoms, $\alpha$ labels layers, and $i, j$ run over the Mn atoms in each layer. With this approach, we generate snapshots of the spin vectors from 5 to $400 \mathrm{~K}$. At low temperature we find that the presence of graphene turns the orientation of the surface spins from mostly in-plane to mostly out of plane (Fig. 3). In passing, we find a bulk $\mathrm{BaMnO}_{3} T_{N}$ of $\sim 100 \mathrm{~K}$, in qualitative agreement with $59 \mathrm{~K}$ from experiment [20]. The bare $\mathrm{BaMnO}_{3}$ slab gives $T_{N} \sim 220 \mathrm{~K}$, which is further raised to $T_{N} \sim 250 \mathrm{~K}$ for the hybrid system (Fig. 4). The largest difference in $T_{N}$ is between bulk and slab, rather than the presence of graphene. These temperatures are very promising for device applications, and suggest a route to both tune surface magnetism with graphene and to make it robust in realistic operating conditions.

\section{TOPOLOGICALLY NONTRIVIAL BANDS AND QUANTIZED ANOMALOUS HALL CONDUCTANCE}

The electronic band structure of the graphene- $\mathrm{BaMnO}_{3}$ interface computed including SOC reveals the opening of two spin-orbit gaps $(\sim 2 \mathrm{meV})$ corresponding to a band crossing near the $\bar{K}$ point, and a gap of $\sim 8 \mathrm{meV}$ at the $\bar{K}$ point, as shown in Fig. 5. These gaps are more than 300 times larger than in pristine graphene, and are a consequence of the interface between graphene and the Mn-terminated $\mathrm{BaMnO}_{3}$ slab. This SIESTA prediction is confirmed on a smaller model system with the all electron FLEUR code: the latter calculations confirm a Rashba splitting near the $\bar{K}$ point, of similar magnitude $(\sim 3.0$ and $10 \mathrm{meV}$ resp.) to those 


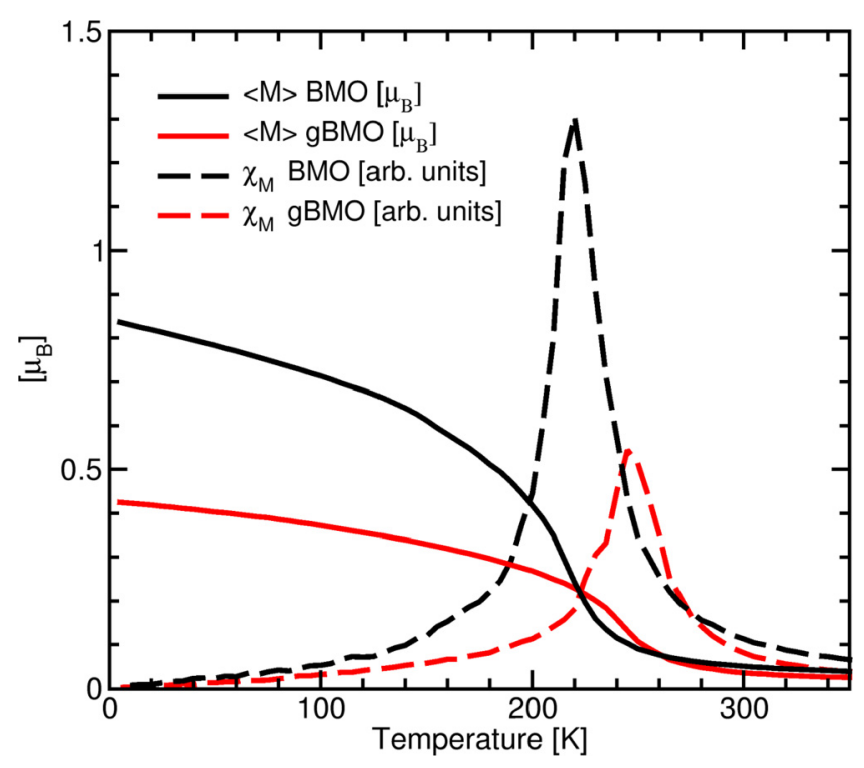

FIG. 4. Average magnetization per atom and magnetic susceptibility for the $\mathrm{BaMnO}_{3}$ and graphene- $\mathrm{BaMnO}_{3}$ slabs. The presence of graphene makes the transition temperature increase from $T_{C}=$ $220 \mathrm{~K}$ (BMO) to $250 \mathrm{~K}$ (gBMO).

found with SIESTA (Fig. 6). This gap is even larger than that observed in graphene on $\mathrm{BiFeO}_{3}$ that shows a similar band structure [27].

The SOC lifts spin degeneracies, suggesting the possibility of a topologically nontrivial band structure. To verify this, we employ Wannier functions [28] and calculate the anomalous

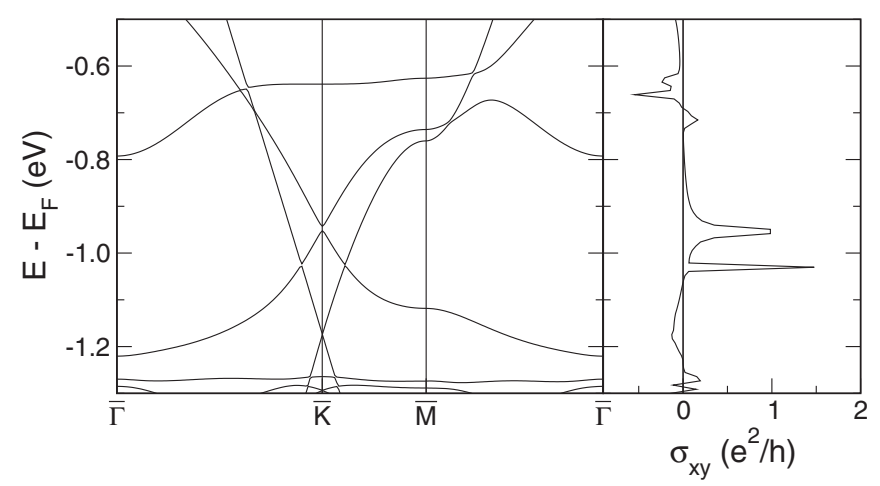

FIG. 6. (Left) Band structure of the 44-atom model system calculated with spin-orbit coupling with the FLEUR code. The Fermi level of the model system $E_{\mathrm{F}}$ was shifted to match the calculations of the entire slab. (Right) Anomalous Hall conductivity as a function of the position of the band filling with respect to $E_{\mathrm{F}}$.

Hall conductivity $\sigma_{x y}$ (AHC), shown as a function of the chemical potential in Fig. 6. A large AHC, close to $2 e^{2} / h$, can be observed at the double SOC gap discussed above. Due to the slightly different dispersion along $\bar{\Gamma}-\bar{K}$ and $\bar{K}-\bar{M}$, and the small offset between the gaps, perfect quantization is not achieved. On the other hand, a 10-meV gap opens in the spin-down Dirac cone, due to the spin-diagonal part of the SOC operator. Here, a robust QAH effect with Chern number +1 develops, consistent with the scenario presented in Ref. [29]. To gain further insight, the wave-vector dependency of the Berry curvature is analyzed. The Berry curvature is localized in the vicinity of $K$ and $K^{\prime}$, so that the presence
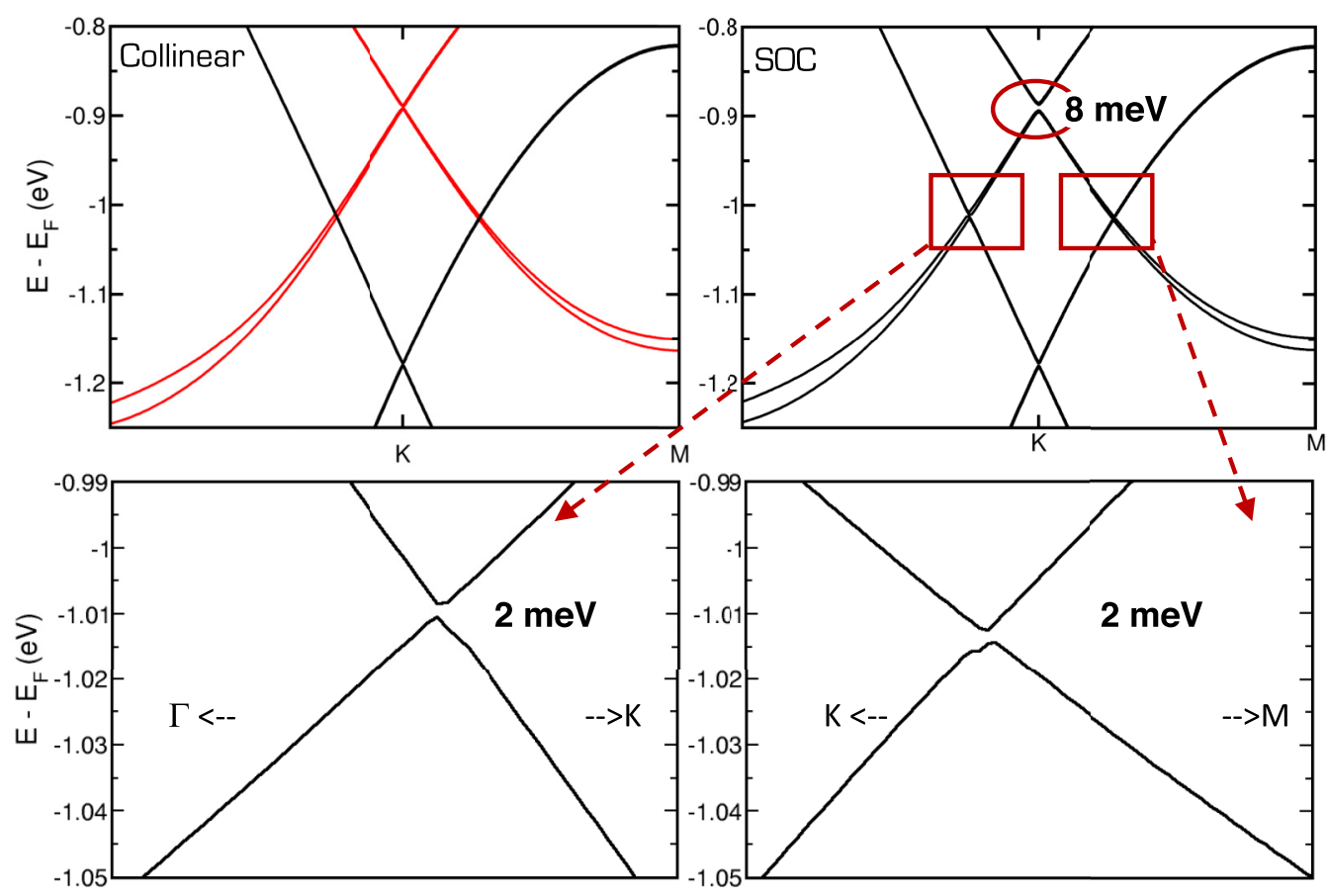

FIG. 5. Electronic band structure of graphene- $\mathrm{BaMnO}_{3}$ computed without ("collinear" spins, top left) and with spin-orbit coupling (top right and bottom panels) with the SIESTA code. In the top left panel, red and black lines denote the electronic bands of majority and minority spins, respectively. Rashba spin-orbit gaps open at band crossing points to the right and left of the $\bar{K}$ point. A $\sim 8$ meV gap opens at the the $\bar{K}$ point due to the spin-diagonal part of the SOC operator. 
(a)

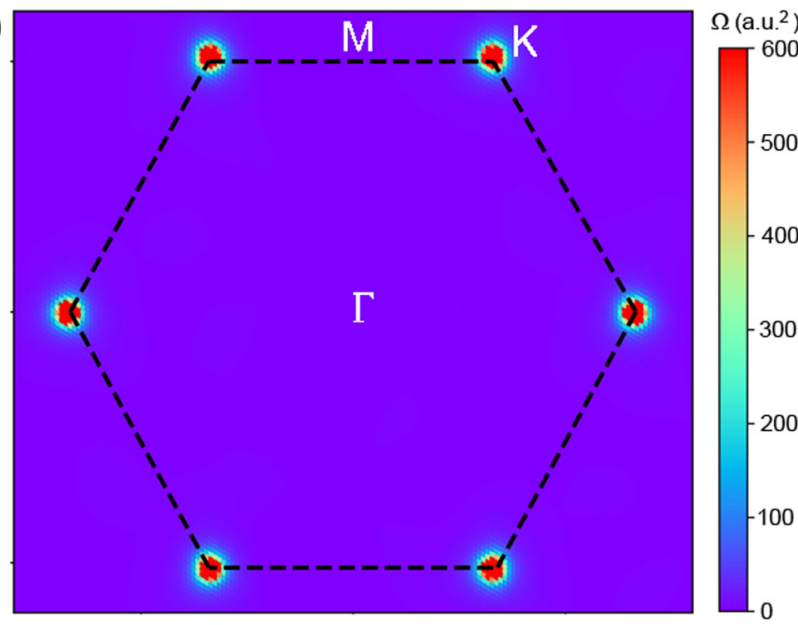

(b)

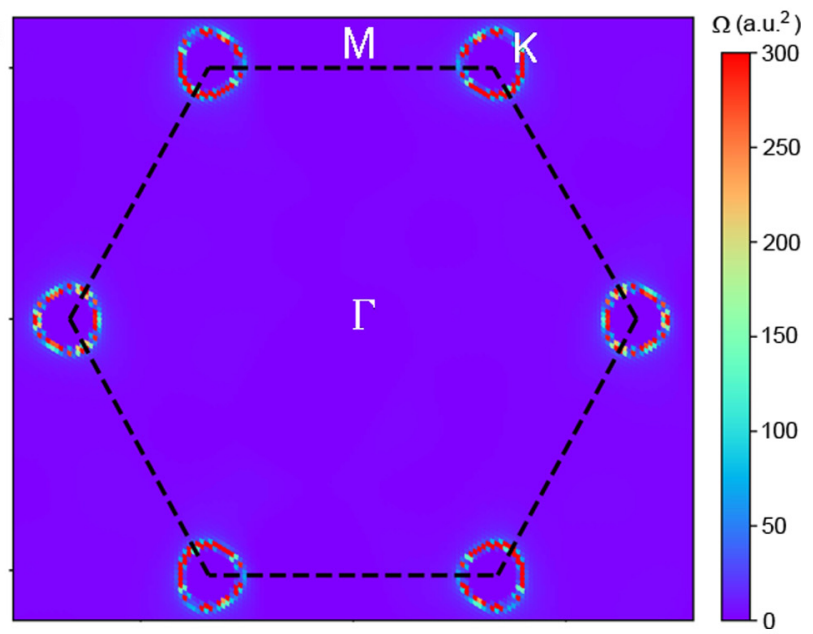

(c)

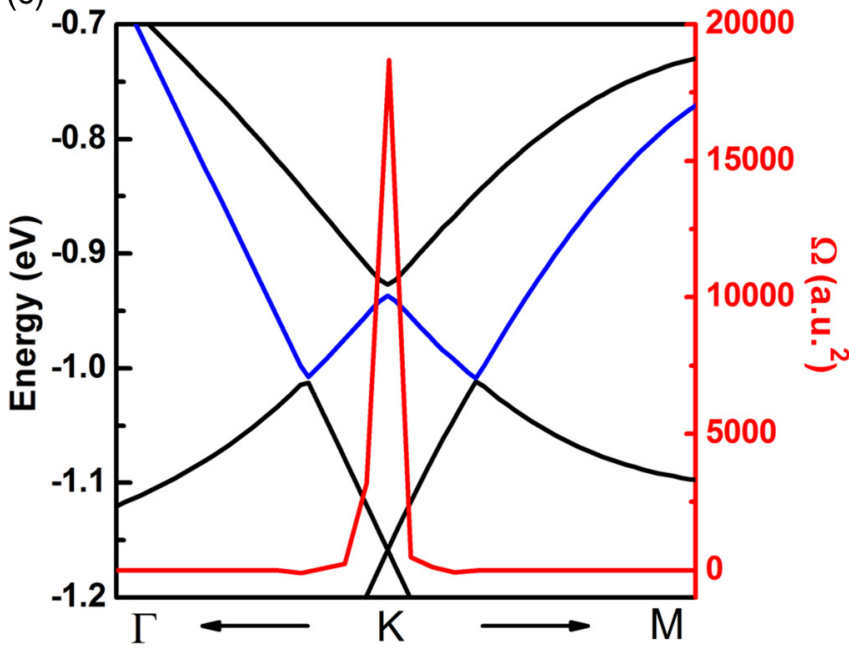

FIG. 7. Berry curvature distribution of the occupied bands at $-0.9 \mathrm{eV}$ (a) and $-1.1 \mathrm{eV}$ (b) in momentum space. It is very localized at the crossing points of the bands, as can be seen in panel (c): The band shown in blue shows a significant contribution to the Berry curvature (red) just around the $K$-point near the gap opening at $-0.9 \mathrm{eV}$.

of other states at the same energy does not destroy the perfect quantization of $\sigma_{x y}$ (Fig. 7). The quantization of the AHC oc-

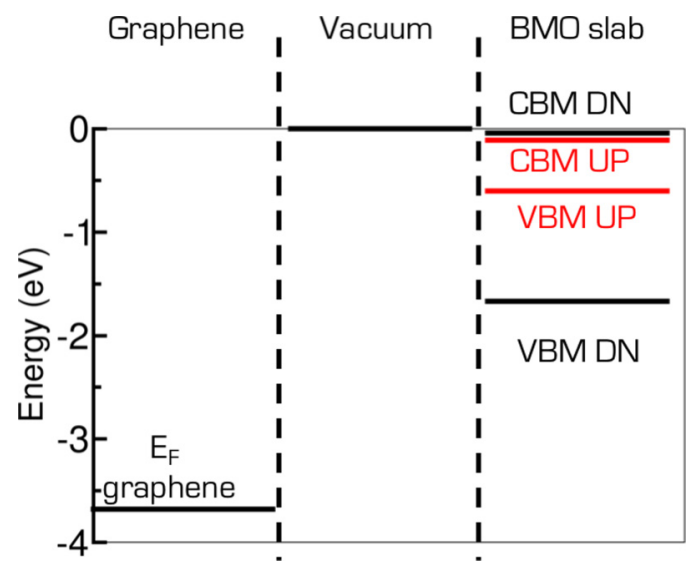

FIG. 8. Schematic representation of the band offset between graphene and $\mathrm{BaMnO}_{3}$ slabs computed from first principles (SIESTA).

curs relatively far from the Fermi level $(-0.9 \mathrm{eV})$ because of the relative electrophilicity of graphene and $\mathrm{BaMnO}_{3}$ (Fig. 8). This energy window could be attained in experiments by doping graphene [10] and/or by applying an external bias. The nontrivial topological properties result from the combination of symmetry breaking at the interface with the triangular arrangement of surface $\mathrm{Mn}$ atoms, analogously to what is predicted for Mn adatoms on graphene [17]. We expect these results to hold for other interfaces that satisfy these conditions; one could also move the topological features closer to the Fermi energy by choosing a substrate which is more electrophilic.

In contrast to previously reported quantized transverse conductivity in graphene on insulators [17,30], the QAHE we observe has a background of "trivial" metallic bands. We dub the Hall effect in graphene- $\mathrm{BaMnO}_{3}$ thin-film hybrid QAHE. The hybrid QAHE in samples with disorder will show a finite anomalous Hall angle, with a robust quantized part added to the disorder-driven AHE of the metallic states visible within the QAHE gap at $\bar{K}$. With increasing disorder, the contribution of the metallic bands to the AHE will decay more rapidly than the quantized part. This will result in an unusual scaling of the hybrid QAHE with temperature and disorder. We anticipate that the presence of geometrically trivial metallic bands at the Fermi energy in hybrid QAH materials may be used to enhance thermal stability or adjust the exchange interactions in the system. This would enable robust QAHE at higher temperatures, a key challenge in current QAHE insulators. With less stringent requirements on the electronic structure, the hybrid QAHE will characterize a much wider classes of 2D spin-orbit materials than those in which the QAHE could be anticipated.

\section{DISCUSSION}

Graphene- $\mathrm{BaMnO}_{3}$ offers an opportunity to combine pronounced nontrivial topological properties, commonly associated with insulators, with metallic magnets. Beyond the hybrid QAHE uncovered here, we will also combine different spin-orbit torques (SOTs) [31]. Recently, it has been demonstrated that topological gaps in 2D ferromagnets lead to colossal "antidamping" torques [32]. These should also 
appear due to the QAH gaps at $\bar{K}$ in graphene- $\mathrm{BaMnO}_{3}$, in addition to fieldlike SOTs mediated by metallic bands. This can provide the unique ability to influence the magnetization of a topologically trivial semiconducting slab, with a small intrinsic magnetoelectric response, by exerting large SOTs at the interface. This is an alternative to the mechanisms recently proposed for antiferromagnetic spintronics [33,34].

\section{CONCLUSIONS}

In conclusion, we have shown that the interface between graphene and the magnetic insulator $\mathrm{BaMnO}_{3}$ is characterized by a strong mutual magnetic interaction. This interaction induces spin polarization in graphene, but also dramatically affects the magnetic substrate, down to several layers below the surface. Hybridization with graphene induces an overall magnetic softening of the $\mathrm{Mn}$ spin interaction, changes the surface layers from AFM to FM, and affects the $\mathrm{BaMnO}_{3}$ easy plane. The electronic band structure of the hybrid system presents a Rashba gap 300 times larger than pristine graphene. The split bands are topologically nontrivial and quantize the anomalous Hall conductivity. These results can be generalized to other C-based nanostructures (nanotubes, nanoribbons, organic molecules), other transition metals, and to more complex heterostructures involving, e.g., transitionmetal dichalcogenides. By exploring different material combinations, one could tune the interaction strength and control the magnetic and topological interface properties. In perspective, these systems enable the design of new functional devices, with optical or electrical control of the hybridization at the interface [1] combining additional degrees of freedom with spin transport.

\section{ACKNOWLEDGMENTS}

The authors acknowledge constructive discussions with J. Varignon and L. Plucinski. Z.Z. acknowledges financial support by the Deutsche Forschungsgemeinschaft (DFG) Grant No. ZA 780/3-1, the Ramon y Cajal MINECO program (Grant No. RYC-2016-19344), the EC H2020-EINFRA-52015 MaX Center of Excellence (Grant No. 676598), the Spanish MINECO (Grant No. FIS2015-64886-C5-3-P), the CERCA programme of the Generalitat de Catalunya (Grant No. 2017SGR1506), and by the Severo Ochoa programme (MINECO, Grant No. SEV-2013-0295). M.J.V. acknowledges funding from the Communauté française de Belgique ARC grant (Grant No. AIMED 15/19-09). Y.M. acknowledges funding from the German Research Foundation (Deutsche Forschungsgemeinschaft), Grant No. MO 1731/5-1. This work has been also financially supported by the Deutsche Forschungsgemeinschaft (DFG) through the Collaborative Research Center SPP 1666. The authors acknowledge computer time from the PRACE-3IP and 4IP on resources Lindgren, Archer, and Salomon (EU Grants No. RI-312763 and No. 653838), CECI, SEGI-ULg and Zenobe hosted by CENAERO (Grant No. GA 1117545), the JARA-HPC projects (No. jara0088, No. JIAS16, and No. JHPC39), the JARA-HPC Vergabegremium and VSR commission on the supercomputer JURECA at Forschungszentrum Jülich.

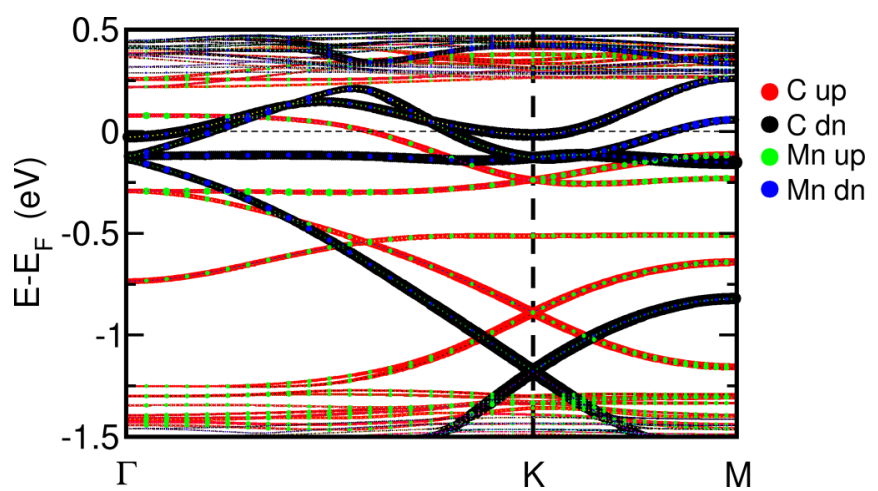

FIG. 9. Electronic band structure of graphene- $\mathrm{BaMnO}_{3}$ projected on the $\mathrm{C}$ and surface $\mathrm{Mn}$ atoms for collinear spins. The spin-up component of $\mathrm{C}$ and $\mathrm{Mn}$ present a considerable hybridization which leads to the Rashba spin-orbit splitting at $\mathrm{K}$. The bands originating from the spin-down components, instead, present a smaller C-Mn hybridization and maintain mostly the graphene character. For this, no gap at $K$ is observed for the corresponding band in SOC calculations.

\section{APPENDIX}

\section{Methods and computational details}

Density functional theory. First-principles calculations have been performed with the SIESTA [21] implementation of density functional theory within the local spin density approximation (LSDA) using the Perdew and Zunger form [35] of the exchange-correlation functional based on Ceperley-Alder data [36]. Calculations were performed assuming both collinear and noncollinear (with spin-orbit coupling) spin alignment. Convergence studies for SIESTA led to a real-space grid cutoff of $1200 \mathrm{Ry}$, a shifted $6 \times 6 \times 12 k$-point sampling of the Brillouin zone, and a Fermi-Dirac smearing of $100 \mathrm{~K}$ in a 30-atom cell for ground-state bulk $\mathrm{BaMnO}_{3}$. Each layer includes $3 \mathrm{Mn}$ atoms. These values ensure an accuracy on total energy differences which is better than $0.5 \mathrm{meV}$ for $\mathrm{BaMnO}_{3}$ bulk (30 atoms), $1.55 \mathrm{meV}$ and $2.6 \mathrm{meV}$ for the slabs without (93 atoms) and with (157 atoms) graphene. The $\mathrm{BaMnO}_{3}$ and graphene- $\mathrm{BaMnO}_{3}$ symmetric slabs consist of $7 \mathrm{Mn}$ layers and $6 \mathrm{BaO}_{3}$ layers. The vacuum between the periodic repetitions of the slabs was larger than $25 \AA$, and the sampling of the Brillouin zone accordingly reduced to $6 \times 6 \times 1$. The graphene layer was fully relaxed (maximal force on atoms $<0.001 \mathrm{eV} / \AA$, maximal stress $<0.01 \mathrm{GPa})$ and the lattice constant of $\mathrm{BaMnO}_{3}$ was strained $(\sim 2 \%)$ to match the $4 \times 4$ graphene supercell. The atomic positions of the $\mathrm{BaMnO}_{3}$ slabs were then relaxed at fixed cell size.

Additionally, DFT calculations have been performed with the full-potential linearized augmented plane-wave method as implemented in the FLEUR code [22]. We performed both LSDA calculations using the calculated lattice parameters taken from Ref. [10] and, for collinear magnetic order also GGA calculations with the experimental lattice parameters from Ref. [20]. In both cases, the in-plane magnetic coupling was found to be small and antiferromagnetic. For the noncollinear magnetic calculations we used a plane-wave cutoff $k_{\max }=3.9$ a.u. and a $5 \times 5 \times 8 k$-point mesh, for the collinear calculations we checked in the values against cutoff values of $k_{\max }=4.3$ a.u. and a $6 \times 6 \times 12$ mesh. To simulate the 

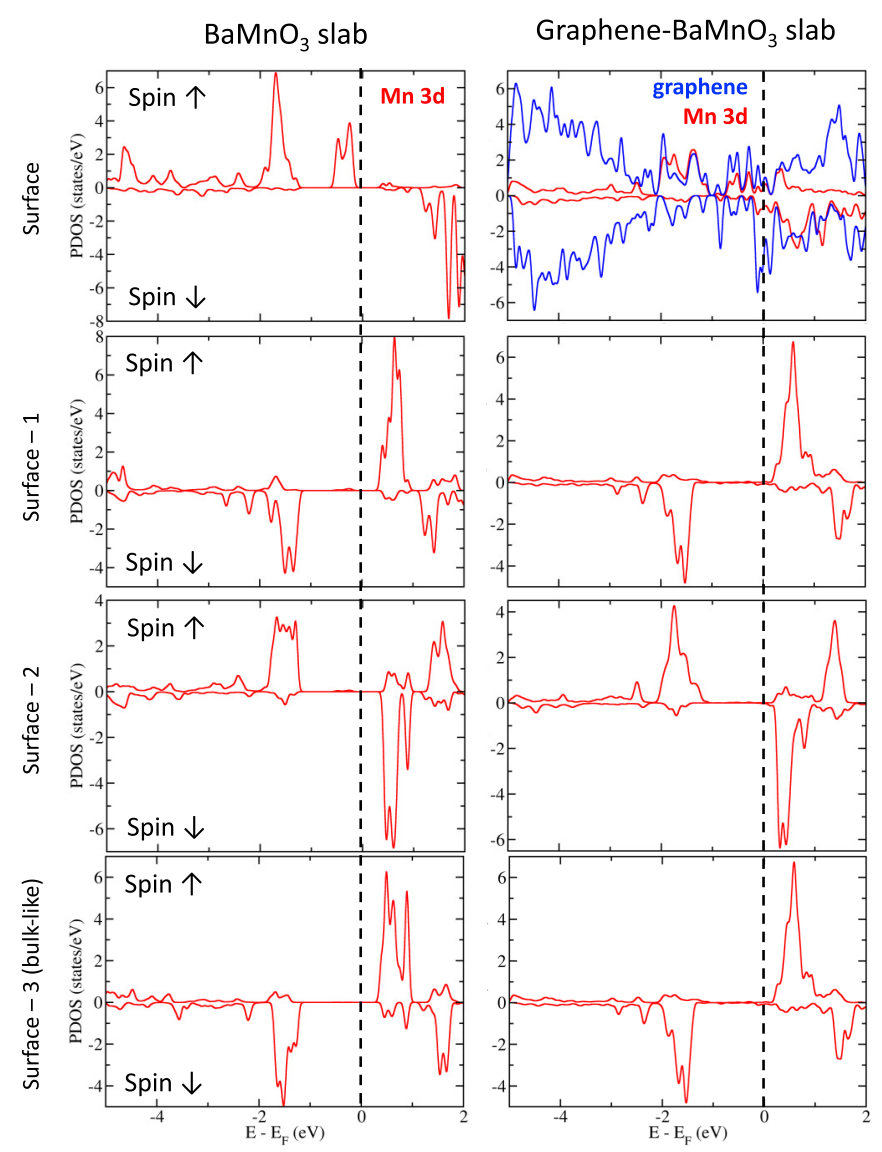

FIG. 10. Projected density of states for the $\mathrm{BaMnO}_{3}$ and graphene- $\mathrm{BaMnO}_{3}$ slabs, for each layer from the surface (top panel) to the center (bottom panel) of the slab computed for collinear spins. Red and blue lines correspond to $\mathrm{Mn} 3 d$ and graphene $2 p$ states, respectively. The PDOS show that Mn $3 d$ states are localized around $E_{F}$ only for the surface layer. This results in an out-of-plane component of the Mn magnetic moment, which is enhanced after interaction with graphene (right panels). As a consequence, the FM in-plane alignment is favored at the surface of the slabs, in contrast with bulk $\mathrm{BaMnO}_{3}$ which is characterized by an AFM in-plane alignment (see Tables I and III).

interface, we use a model system for the graphene-covered surface consisting of the atoms at the interface, i.e., a $4 \times 4$ supercell of graphene and three Mn formula units underneath.
The nine oxygen atoms bonded below the BMO slab were substituted by fluorine to simulate a semi-infinite chemical termination of the slab. A plane-wave cutoff of $k_{\max }=4.3 \mathrm{a}$.u. was used, and a $5 \times 5 k$-point sampling.

Monte Carlo simulations. The Monte Carlo calculations were based on the Metropolis algorithm in a supercell extending $15 \times 15$ primitive vectors in the lateral directions and having a thickness that includes $7 \mathrm{Mn}$ atomic layers. At each temperature, 100000 sampling lattice sweeps were performed, while between subsequent sampling sweeps 5 lattice sweeps were allowed for decorrelation. The calculations were based on the Heisenberg model with nearest-neighbor in-plane and interplane interactions.

To compute the transition temperature in the slab configuration we used Monte Carlo calculations with a periodic supercell consisting of up to $40 \times 40 \mathrm{Mn}$ atoms in the lateral direction and $7 \mathrm{Mn}$ layers. The nearest-neighbor Heisenberg model was treated by the Metropolis algorithm using 25000 thermalizing lattice sweeps and 500000 sampling lattice sweeps at each temperature, starting from 350 to $5 \mathrm{~K}$ with steps of $5 \mathrm{~K}$. Subsequent sampling sweeps were interrupted by 5 lattice sweeps for decorrelation. Figure 4 shows the result for the magnetization and susceptibility, averaged per atom over the full supercell (7 layers), for the cases with and without graphene (gBMO and BMO, respectively). The average magnetic moments are defined as

$$
\langle M\rangle=\frac{1}{7 \mu_{B}} \sum_{i=1}^{7} m_{i} .
$$

The transition temperatures shown in Fig. 4 are dominated by the surface behavior since the weaker bulk exchange interaction parameters (Table III) result in a bulk transition temperature $(\sim 100 \mathrm{~K})$ much lower than the surface one, and since the film is only 7 layers thick.

The MC results demonstrate a crossover and loss of shortrange order at $250 \mathrm{~K}$ for $\mathrm{gBMO}$ and $220 \mathrm{~K}$ for $\mathrm{BMO}$. This should not be strictly interpreted as a phase transition as, for the Mermin-Wagner theorem, there can be no phase transition in the Heisenberg model in two dimensions if the interactions are finite ranged. For this reason, we speak of a transition temperature and not of a critical temperature for the slab case.

Tests with supercells of $20 \times 20$ and $40 \times 40$ primitive vectors in the lateral direction showed the same behavior,

TABLE II. Spin configurations for the computation of the exchange parameters $J_{\|}$and $J_{\perp}$ for bulk $\mathrm{BaMnO}_{3}$ and the corresponding total energies $\left(E_{\mathrm{Tot}}\right.$, in $\mathrm{eV}$, with respect to spin state iv) obtained from first principles. The calculations were performed for bulk $\mathrm{BaMnO}_{3}$ either relaxed in the $P 6_{3} \mathrm{~cm}$ phase or strained to the $4 \times 4$ graphene cell.

\begin{tabular}{|c|c|c|c|c|c|c|c|c|c|c|}
\hline Config & \multicolumn{3}{|c|}{ Layer 1} & \multicolumn{3}{|c|}{ Layer 2} & \multicolumn{3}{|c|}{$E_{\mathrm{Tot}}(\mathrm{eV})$} & Comments \\
\hline ii & - & + & + & - & - & - & 0.541 & 0.570 & 0.672 & $\begin{array}{c}\text { Frustrated AFM in layer } 1 \text {, } \\
\text { FM in layer } 2\end{array}$ \\
\hline iii & + & + & + & - & - & - & 0.015 & 0.023 & 0.026 & $\begin{array}{c}\text { FM in plane, } \\
\text { AFM between planes }\end{array}$ \\
\hline
\end{tabular}


TABLE III. The exchange coupling strengths between Mn atoms in plane $\left(J_{\|}\right)$and between planes $\left(J_{\perp}\right)$ for $\mathrm{BaMnO}_{3}$ bulk (relaxed ground-state structure) computed with SIESTA, FLEUR, and compared with calculations performed with the B1-WC hybrid functional [37]. Units are meV. The fit of the Heisenberg model is performed on three different magnetic configurations (ii, iii, iv), except SIESTA-all (all configurations) and SIESTA-Varignon and the hybrid calculations (which use i, iii, iv).

\begin{tabular}{lcc}
\hline \hline Relaxed & $J_{\|}$ & $J_{\perp}$ \\
\hline FLEUR & -1.08 & -164.8 \\
SIESTA-3 & -0.96 & -139.50 \\
SIESTA-all & -1.00 & -140.55 \\
SIESTA-Varignon & -0.14 & -140.60 \\
Hybrid [37] & -0.14 & -32.77 \\
\hline \hline
\end{tabular}

except at $T=0$, where spurious domains appeared in the spin structure because the supercell dimensions became incommensurate with the noncollinear wavelength of 3 in-plane primitive vectors.

Hall conductivity and Berry curvature. The anomalous Hall conductivity is defined as $\sigma_{x y}=\left(e^{2} / \hbar\right) \mathcal{C}$, where $\mathcal{C}$ is the first Chern number:

$$
\mathcal{C}=\frac{1}{2 \pi} \int_{\mathrm{BZ}} \Omega(\mathbf{k}) d^{2} k
$$

$\Omega(\mathbf{k})$ is the Berry curvature of all occupied bands:

$$
\Omega(\mathbf{k})=\sum_{n, m \in \text { occ }} \sum_{m \neq n} 2 \operatorname{Im} \frac{\left\langle\psi_{n \mathbf{k}}\left|v_{x}\right| \psi_{m \mathbf{k}}\right\rangle\left\langle\psi_{m \mathbf{k}}\left|v_{y}\right| \psi_{n \mathbf{k}}\right\rangle}{\left(\varepsilon_{m \mathbf{k}}-\varepsilon_{n \mathbf{k}}\right)^{2}},
$$

where $m, n$ are band indices, $\psi_{m / n \mathbf{k}}$ and $\varepsilon_{m / n \mathbf{k}}$ are the Bloch wave functions and the corresponding eigenenergies of band $m / n$, respectively, and $v_{x / y}$ are the Cartesian components of the velocity operator.

\section{2. $\mathrm{BaMO}_{3}$ and graphene- $\mathrm{BaMnO}_{3}$ slab: Collinear spin}

The presence of surface states (two localized bands below $E_{F}$ ) on the $\mathrm{BaMnO}_{3}$ slab is revealed by collinear spin calculations of the collinear electronic band structure projected on $\mathrm{C}$ and $\mathrm{Mn}$ atoms (Fig. 9), and corresponding density of states decomposed by layer (Fig. 10 surface states in top left panel). Interfacing $\mathrm{BaMnO}_{3}$ with graphene results in an hybridization between $\mathrm{C}$ and Mn surface states (Fig. 10, top right panel).

\section{Exchange parameters}

We perform collinear spin calculations for a number of independent spin configurations of the $\mathrm{Mn}$ atoms. The $\mathrm{BaMnO}_{3}$ bulk was computed with SIESTA and FLEUR, and slabs with and without graphene using SIESTA. We obtain in each case an overdetermined system of equations and solve it for the

TABLE IV. Spin configurations for the computation of the exchange parameters for the $\mathrm{BaMnO}_{3}$ and graphene- $\mathrm{BaMnO}_{3}$ slabs and the corresponding total energy $\left(E_{\mathrm{Tot}}\right)$ in $\mathrm{eV}$ as obtained with SIESTA. Top and bottom energies refer to the $\mathrm{BaMnO}_{3}$ and graphene-BaMnO${ }_{3}$ slab, respectively. The spin configurations are ordered from the lowest to the highest total energy for the $\mathrm{BaMnO}_{3}$ case. Note that the energetic ordering of the various spin configurations changes after interaction with graphene. The fit of the Heisenberg model has been done using 10 configurations, excluding the two configurations at higher energy for each system. The latter are indicated in bold characters in the table.

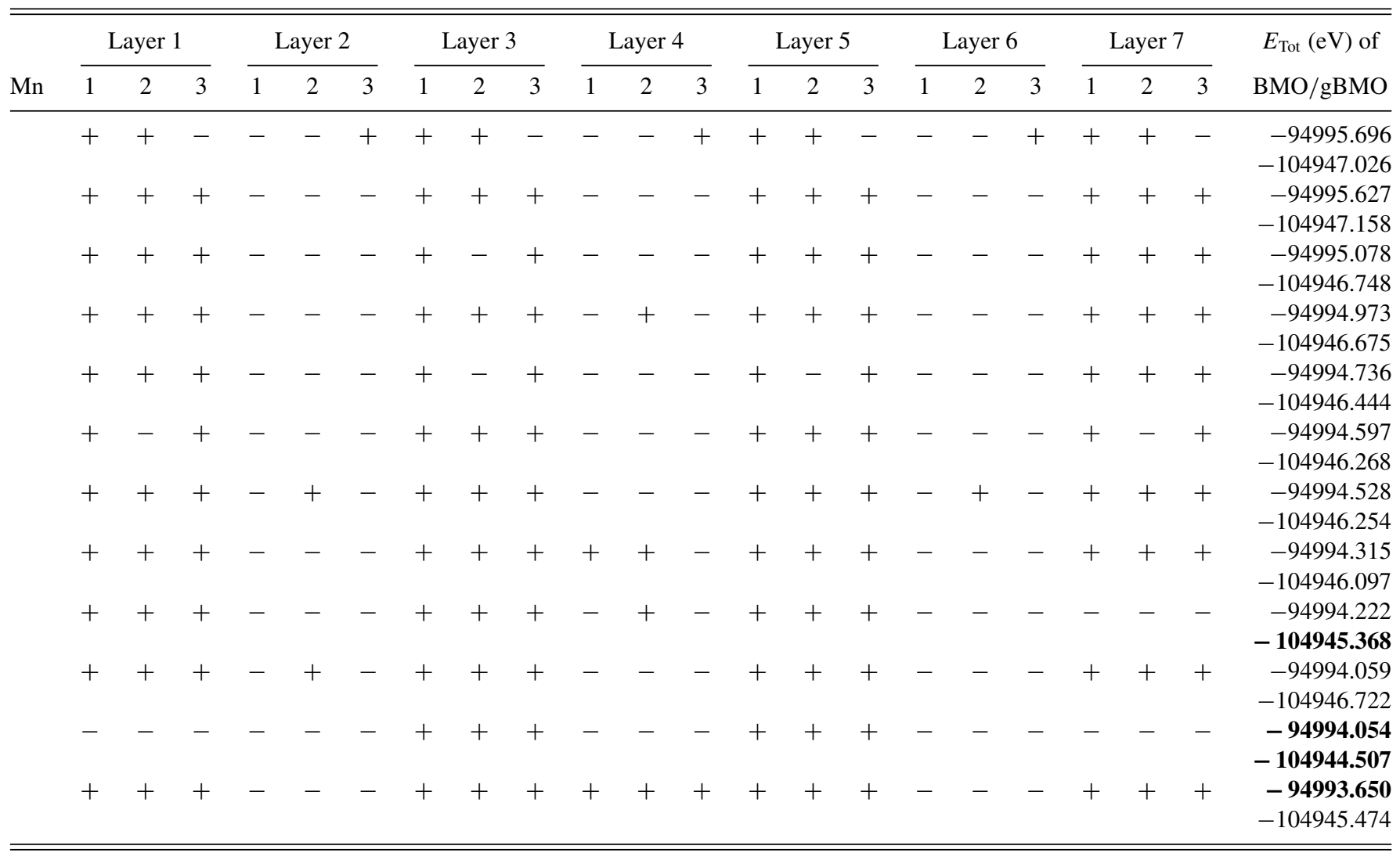


TABLE V. Total energy difference $(\Delta E$, in $\mathrm{meV})$ and total energy difference per $\mathrm{Mn}$ atom $(\Delta E / \mathrm{Mn}$, in meV) for the low-energy spin configurations of $\mathrm{BaMnO}_{3}$ and graphene- $\mathrm{BaMnO}_{3}$ slabs. The destabilization of the in-plane $120^{\circ}$ in the gBMO is due to the surface's preference for a FM alignment, which wins over the bulk triangular arrangement of Mn spins.

\begin{tabular}{lrrrrr}
\hline \hline & \multicolumn{2}{c}{$\mathrm{BaMnO}_{3}$} & & \multicolumn{2}{c}{$\mathrm{gBaMnO}_{3}$} \\
\cline { 2 - 3 } \cline { 5 - 6 } & $\Delta E$ & $\Delta E / \mathrm{Mn}$ & & $\Delta E$ & $\Delta E / \mathrm{Mn}$ \\
\hline FM along $z$ & 47.58 & 2.27 & & 3.47 & 0.17 \\
In-plane FM & 45.10 & 2.15 & & 0.00 & 0.00 \\
In-plane 120 & 0.00 & 0.00 & & 206.60 & 9.84 \\
\hline \hline
\end{tabular}

exchange coupling parameters $J$ using a singular value decomposition. These $J$ are used in a Monte Carlo model to predict the spin dynamics at finite temperature.

Bulk $\mathrm{BaMnO}_{3}$. The Heisenberg model for the bulk material is characterized by two exchange coupling parameters related to the magnetic interaction in plane $\left(J_{\|}\right)$and between adjacent planes $\left(J_{\perp}\right)$. With both $a b$ initio codes we find that the AFM coupling between planes is the dominant effect ( $J_{\perp}$ is negative and several orders of magnitude larger than $J_{\|}$). The in-plane interaction is a frustrated AFM: the collinear spin model cannot reproduce the triangular alignment observed in SOC calculations and in experiment [20], but the resulting Heisenberg Hamiltonian is consistent with both.

The exchange parameters $J_{\|}$and $J_{\perp}$ (and a reference nonmagnetic energy) are obtained by solving a system of (at least) three equations for the total energy of different spin configurations (labeled ii, iii, and iv in Table II) for bulk $\mathrm{BaMnO}_{3}$. The total energies have been obtained from first-principles calculations using a collinear spin arrangement. The unit cell of $\mathrm{BaMnO}_{3}$ was strained to the $4 \times 4$ cell of graphene, in order to allow for a comparison with the exchange parameter obtained for the slab calculations. The atomic positions were relaxed in the configuration iii, characterized by a FM alignment in plane and AFM alignment between planes. These coordinates were then used for the other spin configurations. Relaxing the coordinates further in each spin state would mix the Heisenberg exchange contribution to the energy with other potential energies reflecting the coupling of spins with atomic displacements, which is beyond the "pure" magnetic Hamiltonian we wish to fit. The Heisenberg Hamiltonian for $\mathrm{BaMnO}_{3}$ bulk is

$$
H=-\frac{1}{2} J_{\|} \sum_{\alpha=1}^{2} \sum_{i \neq j} S_{i \alpha} S_{j \alpha}-\frac{1}{2} J_{\perp} \sum_{i} S_{i, 1} S_{i, 2} .
$$

SIESTA and FLEUR provide similar results for $J_{\|}$and $J_{\perp}$. We obtain the same qualitative picture as the values reported in [37], which are obtained with the CRYSTAL code and hybrid exchange correlation functional (Table III), but with harder $J$ by a factor roughly 5 . There are two possible reasons for the discrepancy: the different choice for the exchange correlation functional, or the choice of the three spin configurations used to compute the $J$ 's. Varignon [38] used the configurations iii, iv and a completely FM configuration (labeled i in Table II), which has a very high total energy with respect to the ground state. As all of the vertical spin chains are maximally frustrated, we find that this may bias the solution of the system of equations in some cases (especially below for the slab systems). To test the latter effect (xc functional), we solve the system of equations again, using the configurations $\mathrm{i}$, iii, and iv with total energies from SIESTA and fully relaxed $\mathrm{BaMnO}_{3}$. We find a change in $J_{\|}$, which suggests it is sensitive to having enough configurations which are low-energy perturbations, but little change in $J_{\perp}$, implying that the difference there is mostly due the density functional employed in the calculations. This is a very important topic for future study, and it is not obvious that hybrid functionals will perform more physically: with enough Hartree-Fock exchange some systems can be pushed (sometimes artificially) to become AFM [24-26], whereas here the opposite occurs: hybrid DFT reduces the AFM exchange. More accurate but computationally demanding methods such as the random phase approximation or ladder resummations will be needed as benchmarks.

Slabs of (graphene-) $\mathrm{BaMnO}_{3}$. As above for bulk BMO, the slab magnetic exchange parameters were fit to a series of total energy calculations with the same (relaxed) geometry and different spin configurations. In this reduced symmetry, more configurations are needed to fit the larger number of independent $J$. The full Hamiltonian is given in the main text.

TABLE VI. $\mathrm{BaMnO}_{3}$ slab: ground-state spin configuration. The $\mathrm{Mn}$ spin vectors are in plane and form a triangular $\left(120^{\circ}\right)$ arrangement [Figs. 1(c) and 1(d)].

\begin{tabular}{|c|c|c|c|c|c|c|c|c|c|}
\hline \multirow{2}{*}{$\frac{\mathrm{Mn}}{1}$} & \multirow{2}{*}{$\frac{\text { Layer }}{1}$} & \multirow{2}{*}{$\frac{\sqrt{\left\langle S^{2}\right\rangle}}{2.113}$} & \multicolumn{3}{|c|}{$\langle(S x, S y, S z)\rangle$} & \multirow{2}{*}{$\frac{\sqrt{\left\langle L^{2}\right\rangle}}{0.070}$} & \multicolumn{3}{|c|}{$\langle(L x, L y, L z)\rangle$} \\
\hline & & & -2.113 & -0.001 & -0.001 & & -0.070 & 0.000 & 0.000 \\
\hline 2 & & 2.112 & 1.068 & 1.822 & 0.000 & 0.073 & 0.037 & 0.063 & 0.000 \\
\hline 3 & & 2.111 & 1.066 & -1.822 & -0.000 & 0.073 & 0.037 & -0.064 & 0.000 \\
\hline 1 & 2 & 1.238 & 1.238 & 0.001 & -0.000 & 0.027 & 0.027 & 0.000 & 0.000 \\
\hline 2 & & 1.231 & -0.591 & -1.080 & -0.000 & 0.025 & -0.012 & -0.022 & 0.000 \\
\hline 3 & & 1.231 & -0.590 & 1.081 & 0.000 & 0.025 & -0.012 & 0.022 & 0.000 \\
\hline 1 & 3 & 1.212 & -1.212 & -0.001 & 0.000 & 0.029 & -0.029 & 0.000 & 0.000 \\
\hline 2 & & 1.207 & 0.575 & 1.062 & 0.000 & 0.029 & 0.014 & 0.025 & 0.000 \\
\hline 3 & & 1.207 & 0.575 & -1.062 & -0.000 & 0.029 & 0.014 & -0.025 & 0.000 \\
\hline 1 & 4 & 1.221 & 1.221 & 0.001 & -0.000 & 0.029 & 0.029 & 0.000 & 0.000 \\
\hline 2 & & 1.219 & -0.580 & -1.072 & -0.000 & 0.029 & -0.014 & -0.026 & 0.000 \\
\hline 3 & & 1.219 & -0.580 & 1.072 & 0.000 & 0.029 & -0.014 & 0.026 & 0.000 \\
\hline
\end{tabular}


TABLE VII. $\mathrm{BaMnO}_{3}$ slab: FM alignment of spin vectors on $\mathrm{Mn}$ atom is along the [0001] direction.

\begin{tabular}{|c|c|c|c|c|c|c|c|c|c|}
\hline 1 & $\begin{array}{c}\text { Layer } \\
1\end{array}$ & $\frac{\sqrt{\left\langle S^{2}\right\rangle}}{2.110}$ & 0.000 & 0.000 & 2.110 & $\frac{\sqrt{\left\langle L^{2}\right\rangle}}{0.078}$ & 0.000 & 0.000 & 0.078 \\
\hline 2 & & 2.114 & 0.000 & 0.000 & 2.114 & 0.075 & 0.000 & 0.000 & 0.075 \\
\hline 1 & 2 & 1.238 & 0.000 & 0.000 & -1.238 & 0.027 & 0.000 & 0.000 & -0.027 \\
\hline 2 & & 1.226 & 0.000 & 0.000 & -1.226 & 0.026 & 0.000 & 0.000 & -0.026 \\
\hline 2 & & 1.210 & 0.000 & 0.000 & 1.210 & 0.029 & 0.000 & 0.000 & 0.029 \\
\hline 3 & & 1.210 & 0.000 & 0.000 & 1.210 & 0.029 & 0.000 & 0.000 & 0.029 \\
\hline 1 & 4 & 1.221 & 0.000 & 0.000 & -1.221 & 0.029 & 0.000 & 0.000 & -0.029 \\
\hline 2 & & 1.218 & 0.000 & 0.000 & -1.218 & 0.029 & 0.000 & 0.000 & -0.029 \\
\hline 3 & & 1.218 & 0.000 & 0.000 & -1.218 & 0.029 & 0.000 & 0.000 & -0.029 \\
\hline
\end{tabular}

Due to the choice of mirror-symmetric paraelectric structures, only half of the Mn layers are independent, and each contributes two exchange parameters, for a total of four in plane and three out of plane $J$.

With an offset energy as a final degree of freedom, a minimum of 8 configurations are necessary. In order to test the robustness of the fit, we performed 12 different calculations, and compared their energies, solving the overdetermined linear system with a singular value decomposition. The influence of high-energy spin configurations in determining the exchange parameters is more pronounced in the slab case: using the 8-10 lowest (in energy) configurations the results are fairly consistent, but adding certain strong perturbations (forcing FM alignment between entire neighboring planes of $\mathrm{Mn}$ ) can scramble the results and flip the sign of different exchange parameters: more configurations are not always better. This type of sensitivity of the exchange $J$ to the choice of configurations is not universal (see above for bulk BMO) but should be checked for systematically. In particular, enforcing fully FM or AFM configurations to a system with the opposite type of ground state probably yields worse results, if the aim is to reproduce low-lying excitations with the resulting Heisenberg model. All of the configurations calculated, and the resulting energies for BMO and graphene-BMO, are presented in Table IV with the energies of "pathological" configurations in bold.

\section{4. $\mathrm{BaMO}_{3}$ and graphene- $\mathrm{BaMnO}_{3}$ slab with $\mathrm{SOC}$}

Calculations with SOC for several different initial spin configurations were performed with SIESTA in order to find the easy direction of magnetization. For each calculation, the atomic coordinates were kept fixed to the collinear-spin ground state, but the spin configuration was relaxed until self-consistency on the electron density matrix was achieved. We found that the AFM coupling between the layers is always present and dominant. The differences mostly concern the relative orientation of the spins in each plane. We found three most relevant (lowest-energy) spin configurations, whose total energies (with respect to the ground state) are collected in Table V below:

(i) in-plane spins oriented at $120^{\circ}$ with respect to each other (like in bulk $\mathrm{BaMnO}_{3}$ );

(ii) FM in plane;

(iii) FM along the [0001] direction.

In the $\mathrm{BaMnO}_{3}$ and graphene- $\mathrm{BaMnO}_{3}$ slabs there are three $\mathrm{Mn}$ atoms per layer, and seven Mn layers. As the slabs are symmetric, we only report the values for the layers 1 (surface) to 4 (middle). Bulk values of the magnetic moment are recovered in the middle of the slab both without (Tables VI and VII) and with graphene (Tables VIII and IX).

From the tabulated values we deduce that there is a large orbital momentum $\sqrt{\left\langle L^{2}\right\rangle}$ developing at the surface of

TABLE VIII. Graphene-BaMnO 3 slab: ground-state spin configuration. The Mn spin vectors are FM in plane [Figs. 1(e) and 1(f)].

\begin{tabular}{|c|c|c|c|c|c|c|c|c|c|}
\hline 1 & $\frac{\text { Layer }}{1}$ & $\frac{\sqrt{\left\langle S^{2}\right\rangle}}{1.364}$ & 1.364 & -0.001 & 0.002 & $\frac{\sqrt{\left\langle L^{2}\right\rangle}}{0.047}$ & 0.046 & 0.003 & 0.007 \\
\hline 2 & & 1.364 & 1.364 & 0.001 & 0.002 & 0.047 & 0.046 & -0.003 & 0.007 \\
\hline 1 & 2 & 1.188 & -1.188 & 0.000 & 0.000 & 0.029 & -0.029 & 0.001 & 0.000 \\
\hline 2 & & 1.188 & -1.188 & 0.000 & 0.000 & 0.029 & -0.029 & -0.001 & 0.000 \\
\hline 2 & & 1.228 & 1.228 & 0.000 & 0.000 & 0.028 & 0.028 & 0.000 & 0.000 \\
\hline 3 & & 1.228 & 1.228 & 0.000 & 0.001 & 0.029 & 0.029 & 0.000 & 0.000 \\
\hline 1 & 4 & 1.211 & -1.211 & 0.000 & 0.000 & 0.028 & -0.028 & 0.000 & 0.000 \\
\hline 2 & & 1.211 & -1.211 & 0.000 & 0.000 & 0.028 & -0.028 & 0.000 & 0.000 \\
\hline 3 & & 1.211 & -1.211 & 0.000 & -0.001 & 0.029 & -0.029 & 0.000 & -0.001 \\
\hline
\end{tabular}


TABLE IX. Graphene-BaMnO 3 slab: the Mn spin vectors present a FM ordering along the [0001] direction [Figs. 1(g) and 1(h)].

\begin{tabular}{|c|c|c|c|c|c|c|c|c|c|}
\hline \multirow{2}{*}{$\frac{\mathrm{Mn}}{1}$} & \multirow{2}{*}{$\begin{array}{c}\text { Layer } \\
1\end{array}$} & \multirow{2}{*}{$\frac{\sqrt{\left\langle S^{2}\right\rangle}}{1.364}$} & \multicolumn{3}{|c|}{$\langle(S x, S y, S z)\rangle$} & \multirow{2}{*}{$\frac{\sqrt{\left\langle L^{2}\right\rangle}}{0.051}$} & \multicolumn{3}{|c|}{$\langle(L x, L y, L z)\rangle$} \\
\hline & & & -0.005 & 0.009 & 1.364 & & 0.007 & -0.012 & 0.049 \\
\hline 2 & & 1.364 & -0.005 & -0.009 & 1.364 & 0.051 & 0.007 & 0.012 & 0.049 \\
\hline 3 & & 1.362 & 0.010 & 0.000 & 1.362 & 0.050 & -0.014 & -0.000 & 0.048 \\
\hline 16 & 2 & 1.188 & 0.001 & -0.002 & -1.188 & 0.031 & -0.000 & 0.000 & -0.031 \\
\hline 17 & & 1.188 & 0.001 & 0.002 & -1.188 & 0.031 & -0.000 & -0.000 & -0.031 \\
\hline 18 & & 1.188 & -0.002 & -0.000 & -1.188 & 0.031 & 0.000 & 0.000 & -0.031 \\
\hline 31 & 3 & 1.228 & 0.001 & -0.001 & 1.228 & 0.028 & 0.000 & -0.000 & 0.028 \\
\hline 32 & & 1.228 & 0.001 & 0.001 & 1.228 & 0.028 & 0.000 & 0.000 & 0.028 \\
\hline 33 & & 1.228 & -0.002 & 0.000 & 1.228 & 0.028 & -0.000 & -0.000 & 0.028 \\
\hline 46 & 4 & 1.211 & -0.001 & -0.000 & -1.211 & 0.028 & 0.000 & 0.000 & -0.028 \\
\hline 47 & & 1.211 & -0.001 & 0.000 & -1.211 & 0.028 & 0.000 & 0.000 & -0.028 \\
\hline 48 & & 1.211 & 0.002 & -0.000 & -1.211 & 0.028 & -0.001 & 0.000 & -0.028 \\
\hline
\end{tabular}

$\mathrm{BaMnO}_{3}$. For both $\mathrm{BaMnO}_{3}$ and graphene- $\mathrm{BaMnO}_{3}$ slabs, the orbital momentum is larger when the spin quantization axis is along the [0001] axis, meaning that surface Mn atoms will preferentially have their spin vector orthogonal to the surface.
[1] M. Cinchetti, V. A. Dediu, and L. E. Hueso, Nat. Mater. 16, 507 (2017).

[2] A. Soumyanarayanan, N. Reyren, A. Fert, and C. Panagopoulos, Nature (London) 539, 509 (2017).

[3] J. Varignon, L. Vila, A. Barthélémy, and M. Bibes, Nat. Phys. 14, 322 (2018).

[4] S. Roche et al., 2D Mater. 2, 030202 (2015).

[5] L. E. Hueso, J. M. Pruneda, V. Ferrari, G. Burnell, J. P. ValdésHerrera, B. Simons, P. B. Littlewood, E. Artacho, A. Fert, and N. D. Mathur, Nature (London) 445, 410 (2007).

[6] W. Han, R. K. Kawakami, M. Gmitra, and J. Fabian, Nat. Nanotechnol. 9, 794 (2014).

[7] Z. Zanolli and J.-C. Charlier, Phys. Rev. B 81, 165406 (2010).

[8] A. Cresti, B. K. Nikolić, J. H. Garcia, and S. Roche, Riv. Nuovo Cimento 39, 587 (2016).

[9] Z. Zanolli and J.-C. Charlier, ACS Nano 6, 10786 (2012).

[10] Z. Zanolli, Sci. Rep. 6, 31346 (2016).

[11] A. Hallal, F. Ibrahim, H. Yang, S. Roche, and M. Chshiev, 2D Mater. 4, 025074 (2017).

[12] J. C. Leutenantsmeyer, A. A. Kaverzin, M. Wojtaszek, and B. J. van Wees, 2D Mater. 4, 014001 (2017).

[13] D. V. Averyanov, I. S. Sokolov, A. M. Tokmachev, O. E. Parfenov, I. A. Karateev, A. N. Taldenkov, and V. G. Storchak, ACS Appl. Mater. Interfaces 10, 20767 (2018).

[14] K. Song, D. Soriano, A. W. Cummings, R. Robles, P. Ordejón, and S. Roche, Nano Lett. 18, 2033 (2018).

[15] A. W. Cummings, J. H. Garcia, J. Fabian, and S. Roche, Phys. Rev. Lett. 119, 206601 (2017).

[16] L. A. Benítez, J. F. Sierra, W. Savero Torres, A. Arrighi, F. Bonell, M. V. Costache, and S. O. Valenzuela, Nat. Phys. 14, 303 (2018).

[17] J. Ding, Z. Qiao, W. Feng, Y. Yao, and Q. Niu, Phys. Rev. B 84, 195444 (2011).

[18] M. Gmitra, S. Konschuh, C. Ertler, C. Ambrosch-Draxl, and J. Fabian, Phys. Rev. B 80, 235431 (2009).

[19] Previous works focused on the interaction between organic molecules and a metallic monolayer $[39,40]$ or bilayer $[41,42]$.
[20] E. J. Cussen and P. D. Battle, Chem. Mater. 12, 831 (2000).

[21] J. M. Soler, E. Artacho, J. D. Gale, A. Garcia, J. Junquera, P. Ordejón, and D. Sánchez-Portal, J. Phys.: Condens. Matter 14, 2745 (2002).

[22] http://www.flapw.de.

[23] T. Moriya, Phys. Rev. 120, 91 (1960).

[24] J. Z. Zhao, W. Fan, M. J. Verstraete, Z. Zanolli, J. Fan, X. B. Yang, H. Xu, and S. Y. Tong, Phys. Rev. Lett. 117, 116101 (2016).

[25] J. Z. Zhao, W. Fan, M. J. Verstraete, Z. Zanolli, J. Fan, X. B. Yang, H. Xu, and S. Y. Tong, Phys. Rev. Lett. 118, 239602 (2017).

[26] S. Lemal, N. Nguyen, J. de Boor, P. Ghosez, J. Varignon, B. Klobes, R. P. Hermann, and M. J. Verstraete, Phys. Rev. B 92, 205204 (2015).

[27] Z. Qiao, W. Ren, H. Chen, L. Bellaiche, Z. Zhang, A. H. MacDonald, and Q. Niu, Phys. Rev. Lett. 112, 116404 (2014).

[28] A. A. Mostofi, J. R. Yates, G. Pizzi, Y.-S. Lee, I. Souza, D. Vanderbilt, and N. Marzari, Comput. Phys. Commun. 185, 2309 (2014).

[29] H. Zhang, F. Freimuth, G. Bihlmayer, M. Ležaić, S. Blügel, and Y. Mokrousov, Phys. Rev. B 87, 205132 (2013).

[30] H. Zhang, C. Lazo, S. Blügel, S. Heinze, and Y. Mokrousov, Phys. Rev. Lett. 108, 056802 (2012).

[31] A. Manchon, I. Miron, T. Jungwirth, J. Sinova, J. Zelezny, A. Thiaville, K. Garello, and P. Gambardella, arXiv:1801.09636.

[32] J. P. Hanke, F. Freimuth, C. Niu, S. Blügel, and Y. Mokrousov, Nat. Commun. 8, 1479 (2017).

[33] L. Smejkal, Y. Mokrousov, B. Yan, and A. MacDonald, Nat. Phys. 14, 242 (2018).

[34] J. Zelezny, P. Wadley, K. Olejnik, A. Hoffmann, and H. Ohno, Nat. Phys. 14, 220 (2018).

[35] J. P. Perdew and A. Zunger, Phys. Rev. B 23, 5048 (1981).

[36] D. M. Ceperley and B. J. Alder, Phys. Rev. Lett. 45, 566 (1980).

[37] J. Varignon and P. Ghosez, Phys. Rev. B 87, 140403(R) (2013).

[38] J. Varignon (private communication). 
[39] M. Callsen, V. Caciuc, N. Kiselev, N. Atodiresei, and S. Blügel, Phys. Rev. Lett. 111, 106805 (2013).

[40] J. Brede, N. Atodiresei, V. Caciuc, M. Bazarnik, A. Al-Zubi, S. Blügel, and R. Wiesendanger, Nat. Nanotechnol. 9, 1018 (2014).
[41] R. Friedrich, V. Caciuc, N. S. Kiselev, N. Atodiresei, and S. Blügel, Phys. Rev. B 91, 115432 (2015).

[42] R. Friedrich, V. Caciuc, N. Atodiresei, and S. Blügel, Phys. Rev. B 92, 195407 (2015). 